JOURNAL OF SYNCHROTRON RADIATION

ISSN 1600-5775

Received 8 July 2016

Accepted 13 October 2016

Edited by M. Weik, Université Grenoble Alpes, France

\# These authors contributed equally to this work.

Keywords: serial synchrotron crystallography; mercury single-wavelength anomalous diffraction; radiation damage.

Supporting information: this article has supporting information at journals.iucr.org/s

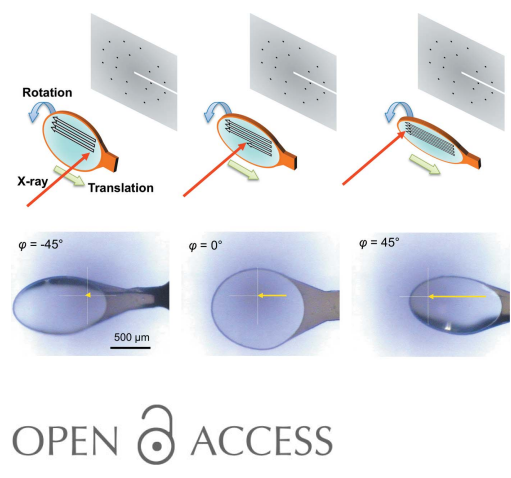

\section{Development of a dose-limiting data collection strategy for serial synchrotron rotation crystallography}

\author{
Kazuya Hasegawa, ${ }^{\mathrm{a}}$ ‡ Keitaro Yamashita, ${ }^{\mathrm{b}} \neq$ Tomohiro Murai, ${ }^{\mathrm{c}}$ Nipawan Nuemket, ${ }^{\mathrm{a}}$ \\ Kunio Hirata, ${ }^{\text {b,d }}$ Go Ueno, ${ }^{b}$ Hideo Ago, ${ }^{b}$ Toru Nakatsu, ${ }^{b, c}$ Takashi Kumasaka ${ }^{a, b}$ and \\ Masaki Yamamoto ${ }^{\mathbf{b}}$ *

\footnotetext{
aJapan Synchrotron Radiation Research Institute, 1-1-1 Kouto, Sayo 679-5198, Japan, ${ }^{\mathbf{b}}$ RIKEN SPring-8 Center, 1-1-1 Kouto, Sayo 679-5148, Japan, 'Graduate School of Pharmaceutical Sciences, Kyoto University, Sakyo, Kyoto 606-8501, Japan, and dapan Science and Technology Agency, Precursory Research for Embryonic Science and Technology (PRESTO), 4-1-8 Honcho, Kawaguchi, Saitama 332-0012, Japan.

${ }^{*}$ Correspondence e-mail: yamamoto@riken.jp
}

Serial crystallography, in which single-shot diffraction images are collected, has great potential for protein microcrystallography. Although serial femtosecond crystallography (SFX) has been successfully demonstrated, limited beam time prevents its routine use. Inspired by SFX, serial synchrotron crystallography (SSX) has been investigated at synchrotron macromolecular crystallography beamlines. Unlike SFX, the longer exposure time of milliseconds to seconds commonly used in SSX causes radiation damage. However, in SSX, crystals can be rotated during the exposure, which can achieve efficient coverage of the reciprocal space. In this study, mercury single-wavelength anomalous diffraction (Hg-SAD) phasing of the luciferin regenerating enzyme (LRE) was performed using serial synchrotron rotation crystallography. The advantages of rotation and influence of dose on the data collected were evaluated. The results showed that sample rotation was effective for accurate data collection, and the optimum helical rotation step depended on multiple factors such as multiplicity and partiality of reflections, exposure time per rotation angle and the contribution from background scattering. For the LRE microcrystals, $0.25^{\circ}$ was the best rotation step for the achievable resolution limit, whereas a rotation step larger than or equal to $1^{\circ}$ was favorable for $\mathrm{Hg}-\mathrm{SAD}$ phasing. Although an accumulated dose beyond 1.1 MGy caused specific damage at the $\mathrm{Hg}$ site, increases in resolution and anomalous signal were observed up to $3.4 \mathrm{MGy}$ because of a higher signal-to-noise ratio.

\section{Introduction}

Macromolecular crystallography (MX) has contributed to the structure determination of more than 109000 proteins as of September 2016 (http://www.wwpdb.org), but there are still many challenging and scientifically important targets yet to have their structure solved. One of the difficulties in structure determination of these targets is obtaining large, well diffracting crystals. To cope with smaller samples, synchrotron microfocus beamlines have been developed (Riekel et al., 2005; Fischetti et al., 2009; Flot et al., 2010; Smith et al., 2012; Hirata et al., 2013). A microbeam with a photon flux density of $10^{10}$ to $10^{11}$ photons $\mathrm{s}^{-1} \mu \mathrm{m}^{-2}$ not only increases the diffraction intensity but also decreases the background scattering from the surrounding mother liquor or buffer.

However, great care is needed when using a microbeam because radiation damage causes diffraction data quality to deteriorate (Holton, 2009; Garman, 2010). Henderson (1990) 
estimated that the diffraction intensity halves after accumulation of $20 \mathrm{MGy}$ during MX data collection at cryogenic temperatures. Owen et al. (2006) experimentally determined a recommended dose limit of $30 \mathrm{MGy}$, at which the diffraction intensity of apoferritin and holoferritin crystals decreased to $70 \%$ of the initial value. They showed that biological information obtained from the structure beyond this limit is compromised. Liebschner et al. (2015) reported that the diffraction intensity of thaumatin crystals decreased to $70 \%$ of the initial value upon accumulation of about $10 \mathrm{MGy}$, and suggested that the dose limit depended on the type of protein crystal. These dose limits force a compromise between attainable resolution and completeness/multiplicity. Radiation damage also affects the local structure of proteins, such as breakage of disulfide bonds and decarboxylation of glutamates and aspartates (Weik et al., 2000; Burmeister, 2000; Ravelli \& McSweeney, 2000). The heavy atoms bound to proteins are especially susceptible to damage because they possess larger photoelectric cross sections than those of lighter atoms. Such local damage is known to affect experimental phasing using heavy atoms (Schiltz et al., 2004; Ramagopal et al., 2005; Ravelli et al., 2005; González, 2007). Damage to local sites progresses faster than global damage and its rate depends on various factors such as the species damaged and the environment of the damaged site (Holton, 2009; Garman, 2010).

One approach to decrease the effect of radiation damage is to translate the crystal during helical data collection, which is applicable when the beam is smaller than the crystal (Flot $e t$ al., 2010). By translating the crystal, fresh crystal volume is exposed to the beam to reduce the overall dose and thus partially mitigate the influence of radiation damage. When the crystal is too small to translate, it is difficult to obtain complete data from one crystal. In these cases, a multicrystal data collection strategy is useful, in which data are completed by merging small wedges collected from multiple crystals. This approach has been automated by combining raster scanning to identify suitable crystal positions and subsequent partial data collection (Zander et al., 2015; Hirata et al., 2017). However, this strategy still has drawbacks; the number of images that can be collected from one crystal is limited when the crystal is small. Moreover, a high dose is needed to identify suitable positions of weakly diffracting crystals, which can lead to severe damage even prior to full data collection.

Serial femtosecond crystallography (SFX) at X-ray freeelectron lasers (XFELs) (Chapman et al., 2011) is another approach based on the principle of 'diffraction before destruction' (Neutze et al., 2000); the pulse duration of a few to a few tens of femtoseconds used in SFX enables collection of a diffraction pattern before the protein structure is destroyed by a Coulomb explosion (Boutet et al., 2012). To date, the performance of SFX in microcrystallography has been exemplified by successful de novo phasing (Barends et al., 2014; Yamashita et al., 2015; Nakane et al., 2015; Fukuda et al., 2016; Nass et al., 2016) as well as structure determination using molecular replacement (Chapman et al., 2011; Boutet et al., 2012; Redecke et al., 2013; Liu et al., 2013; Weierstall et al.,
2014; Kern et al., 2014; Kupitz et al., 2014; Tenboer et al., 2014). However, the limited availability of XFEL beam time prevents SFX from becoming a general method for protein microcrystallography. Moreover, with the current technique, a huge number of diffraction patterns are required for accurate data collection. For example, single-wavelength anomalous diffraction (SAD) phasing using a gadolinium derivative of lysozyme required 60000 indexed patterns (Barends et al., 2014), which was recently lowered to 7000 by an improvement in the data processing (Nass et al., 2016).

Inspired by the success of SFX, serial synchrotron crystallography (SSX) has been examined at synchrotron MX beamlines (Gati et al., 2014; Stellato et al., 2014; Nogly et al., 2015; Botha et al., 2015; Coquelle et al., 2015). Unlike in SFX, the exposure time of milliseconds to seconds in SSX can cause radiation damage; however, it has the advantage that the crystals can be rotated during exposure, which increases the accuracy and efficiency of data collection (Huang et al., 2015, 2016). Gati et al. (2014) collected diffraction data from loopharvested microcrystals of $T b C$ atB by two-dimensional raster scanning combined with goniometer rotation. They solved the structure by molecular replacement and demonstrated the capability of serial synchrotron rotation crystallography (SSROX) for protein microcrystallography with tolerable radiation damage. Inspired by their work, here we report the $\mathrm{Hg}$ SAD phasing of luciferin regenerating enzyme (LRE) using SS-ROX. To demonstrate the prospects and limitations of serial microcrystallography at synchrotron beamlines, we evaluate the effects of rotation angle per image on resolution and data quality, and verify the influence of dose on the anomalous signal and phasing. Unlike Gati et al. (2014), rather than grouping and processing together consecutive images from the same crystal, we processed all the images as random snapshots. Our results show that sample rotation is effective for accurate data collection. Larger rotation steps enable $\mathrm{Hg}$ SAD phasing with fewer images, but the optimum rotation step depends on the balance between the multiplicity and partiality of reflections, exposure time per rotation angle, and the contribution from background scattering. Although there is specific damage at the $\mathrm{Hg}$ site when the accumulated dose exceeds $1.1 \mathrm{MGy}$, increases in resolution and anomalous signal intensity are observed up to $3.4 \mathrm{MGy}$ because of a higher signal-to-noise ratio $(\mathrm{S} / \mathrm{N})$.

\section{Material and methods}

\subsection{Sample preparation}

Microcrystals of mercury-bound LRE consisting of 308 amino acid residues were used. The crystals belonged to the space group $P 2_{1} 2_{1} 2_{1}$ with unit-cell parameters $a=47.3, b=$ 76.7, $c=84.0 \AA$, and contained one monomer per asymmetric unit. There was one major and one minor mercury site in the vicinity of Cys52. Yamashita et al. (2015) have previously described the protocol for the preparation of LRE microcrystals and their mercury derivative. The LRE microcrystals were rod shaped with a thickness of 3-5 $\mu \mathrm{m}$ and length of 20 
$50 \mu \mathrm{m}$. The LRE microcrystals were mounted on LithoLoops (Protein Wave Co., Japan) with a diameter of $1.0 \mathrm{~mm}$ and flash cooled under a cryo stream operating at $107 \mathrm{~K}$. The average thickness of the sample at the center of a loop was $0.12 \mathrm{~mm}$ (0.02 r.m.s.d.).

\subsection{Data collection with SS-ROX}

Data collection was conducted at SPring-8 BL41XU using a wavelength of $0.9839 \AA$, which was the same wavelength as that used during a SFX experiment at SACLA (Yamashita et al., 2015, 2017). A microbeam of $5 \mu \mathrm{m}$ (vertical; V) $\times 4 \mu \mathrm{m}$ (horizontal; $\mathrm{H}$ ) (full width at halfmaximum) with a photon flux of $2.2-2.4 \times 10^{12}$ photons s $^{-1}$ was used. The samples were kept at $107 \mathrm{~K}$ under a cryo nitrogen flow using CRYOCOOL-G2b-LT (Cryo Industries of America, Inc., USA). Diffraction images were recorded with a PILATUS3 6M detector (Dectris Ltd, Switzerland).

The loop on which the crystals were loaded was rasterscanned with rotation of the goniometer spindle axis (Fig. 1). The spindle was rotated as the crystal was being translated. An area of $750 \mu \mathrm{m}(\mathrm{V}) \times 720 \mu \mathrm{m}(\mathrm{H})$ on the loop was scanned by 75 horizontal helical scans with a vertical interval of $10 \mu \mathrm{m}$ between neighboring scans. A total of 180 images were recorded in each horizontal helical scan with a horizontal translation of $4 \mu \mathrm{m}$ between each image. This translation length was identical to the beam width. The rotation and exposure time per image were changed as described below.

2.2.1. Data collection using different helical rotation steps. To examine the influence of the helical rotation step on the data, we collected seven datasets using a helical rotation step per image $(\Delta \varphi)$ of $0.0^{\circ}, 0.1^{\circ}, 0.25^{\circ}, 0.5^{\circ}, 1.0^{\circ}, 1.5^{\circ}$ and $2.0^{\circ}$; these datasets are labeled Rs-0, Rs-0.1, Rs-0.25, Rs-0.5, Rs-1, Rs-1.5 and Rs-2, respectively. The total goniometer rotation range for each helical scan was $\pm 0^{\circ}$ for Rs $-0, \pm 9^{\circ}$ for Rs- $0.1, \pm 22.5^{\circ}$ for Rs- 0.25 and $\pm 45^{\circ}$ for Rs-0.5, where $\varphi=0^{\circ}$ is the angle where the loop surface is perpendicular to the incident beam. For Rs-1, to prevent the scan range from being $\pm 90^{\circ}$, the scan area was divided into two areas of $750 \mu \mathrm{m}(\mathrm{V}) \times 360 \mu \mathrm{m}(\mathrm{H})$ and the number of images per helical line was halved. This decreased the scan range to $\pm 45^{\circ}$. In a similar way, the scan areas of both Rs-1.5 and Rs-2 were divided into three and four, respectively, and each area was scanned for a rotation of $\pm 45^{\circ}$. All datasets were collected with a frame rate of 50 frames s$^{-1}$, which corre-
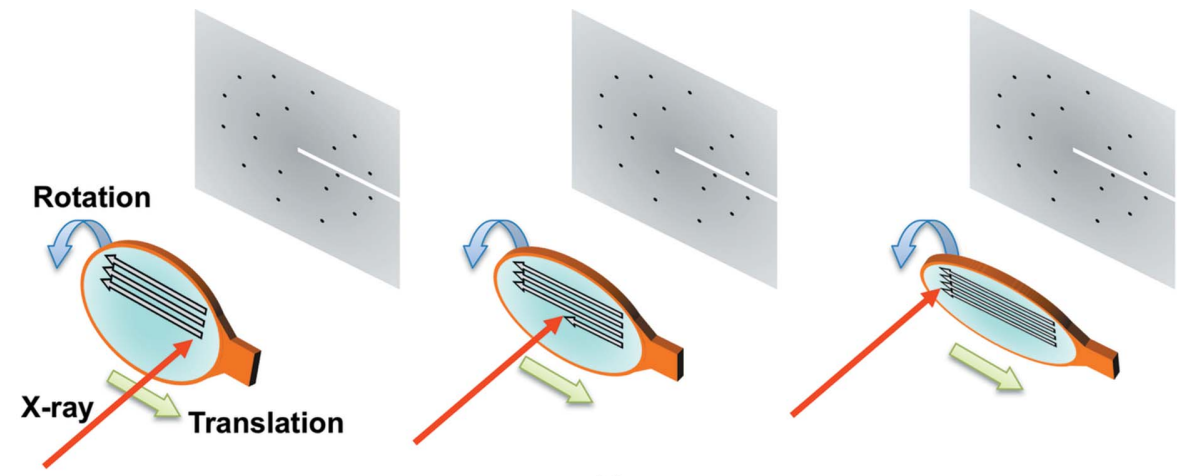

(a)
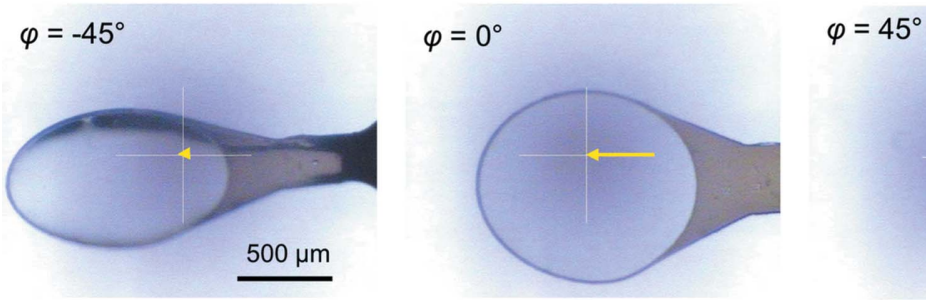

(b)

Figure 1

Overview of SS-ROX data collection. (a) Schematic diagrams of two-dimensional raster scans with goniometer rotation. The loop on which the crystals were loaded was raster-scanned with rotation of the goniometer spindle axis. (b) Photographs of a horizontal helical scan; yellow arrows represent relative movement of the incident X-ray position. $\varphi$ is the goniometer spindle angle. 
helical scan that completely went across a crystal was simulated in the calculation. Photon fluxes of $2.4 \times 10^{12}$ and $2.3 \times 10^{12}$ (photons s $^{-1}$ ) were used for Da-1.3 to Da-26 and Ds-1. $1^{i \text { th }}(i=1,2, \ldots, 25)$, respectively. Among several dosebased metrics calculated by RADDOSE-3D, we used the average dose-exposed region (AD-ER) (Zeldin et al., 2013) as a metric for the absorbed dose. The accumulated dose for each crystal after data collection is equal to the estimated value. However, this is an expected maximum dose per frame because there is a possibility that the X-rays started to hit the crystal in the middle of the exposure of a frame and the crystal was not totally bathed in the beam at the beginning of the exposure.

\subsection{Data processing and Hg-SAD phasing}

2.3.1. Hit finding. We adapted the peak finding function of Cheetah (Barty et al., 2014) for hit finding. In peak finding, integrated intensity was evaluated using background based on radial average. An image was considered a hit if three or more spots were found in the area with resolution lower than $5.0 \AA$. The hit images were subjected to data processing using CrystFEL (White et al., 2016) and XDS (Kabsch, 2010b). The modified version of Cheetah (cheetah.local_singles) is available on GitHub (https://github.com/keitaroyam/cheetah/tree/ eiger-zmq).

2.3.2. Data processing using CrystFEL. We used CrystFEL (White et al., 2016) version 0.6.1 to index, integrate and merge data. Prior to data processing, PILATUS cbf files were converted to the hdf5 format. The sensor gaps were removed in this conversion. Diffraction spots were selected using the built-in zaef algorithm (Zaefferer, 2000) and indexed using DirAx (Duisenberg, 1992). Spot intensities were integrated using direct summation; peak intensity was estimated within a radius of two pixels, and background was estimated with radii between four and six pixels. First, we processed 200 randomly selected images and optimized their zaef parameters by maximizing the number of indexed patterns, which resulted in a threshold and minimum gradient of 50 and 20000, respectively. The zaef parameter 'minimum SNR' was fixed to 1 . Using this result, we averaged the refined detector shifts and applied the averaged value to the sensor geometry configuration file. These parameters were then used to process all images. The intensities in the stream files were merged using the Monte Carlo method with per-frame scaling using the process_hkl scale.

Because all images were rotational snapshots except for the still dataset (Rs-0), Lorentz-factor correction with respect to the spindle axis was required. We applied the inverse Lorentz factor $\left|\mathbf{m}_{2} \cdot\left(\mathbf{S} \times \mathbf{S}_{0}\right)\right| /\left(|\mathbf{S}| \cdot\left|\mathbf{S}_{0}\right|\right)$, where $\mathbf{m}_{2}$ is the spindle axis (unit vector) and $\mathbf{S}$ and $\mathbf{S}_{0}$ are the wavevectors of the diffracted and incident beam, respectively (Kabsch, 2010a), to intensities and estimated errors in the stream file. Intensities were merged as described above.

2.3.3. Data processing using XDS. We used the custommade script kamo.single_images_integration to process random snapshots using XDS (Kabsch, 2010b) (version of 15 October 2015). An overview of this data processing is given in Fig. S1 of the supporting information. The images were processed individually. Indexing was performed first without using known unit-cell parameters as prior information. If indexing was not successful or not consistent with the known unit cell, then it was performed again including prior information. If the new result was not consistent with the known unit cell, the image processing was considered a failure. Next, integration by three-dimensional (3D) profile fitting was performed. All integrated intensities were written to file (MINPK=0). Because the intensities in this file were 'full' intensities estimated from 3D profiles, partial intensities were put aside to be stored in another file for evaluation by multiplying intensities and estimated errors by the percentage of observed reflection intensity (PEAK) as reported by $X D S$. The integrated data were subjected to a CORRECT job without any empirical correction or refinement of geometric parameters. Only non-empirical corrections including a polarization correction, and an absorption correction for air and for the Si sensor were applied in the CORRECT job. To prevent rejection of reflections where the centroids in the rotation direction were estimated to be located far away from the image number, filenames of data_10000.cbf were always used and DATA_RANGE $=120000$ was specified in CORRECT. 'Full' and 'partial' intensities were saved separately to decide later which gave a better result.

Integrated intensities (full and partial reflections separately) were merged using the custom-made script kamo. merge_single_images_integrated, which merged intensities and estimated the error in a Monte Carlo manner with frame scaling using the following equations as process_hkl in CrystFEL (White et al., 2012),

$$
\begin{gathered}
\langle I(h k l)\rangle=\frac{1}{N} \sum_{j=1}^{N} I_{j}(h k l), \\
\sigma^{2}[\langle I(h k l)\rangle]=\frac{1}{N^{2}} \sum_{j=1}^{N}\left[I_{j}(h k l)-\langle I(h k l)\rangle\right]^{2},
\end{gathered}
$$

where $I_{j}(h k l)$ is the $j$ th observation of the reflection intensity and $\langle I(h k l)\rangle$ is the merged intensity obtained by Monte Carlo integration. The per-frame linear scale factor for the $i$ th frame $q_{i}$ was calculated to minimize the squared residual between $q_{i} I^{i}(h k l)$ and $\langle I(h k l)\rangle$, where $I^{i}(h k l)$ is the observed intensity for the $i$ th frame. In this script, the minimum PEAK value (like $\mathrm{MINPK}=$ in $X D S$ ) can be specified. The randomly halved datasets were prepared for calculation of $\mathrm{CC}_{1 / 2}, \mathrm{CC}_{\mathrm{ano}}$ and other parameters. The statistics were calculated using compare_hkl and check_hkl in CrystFEL. Note that we used $X D S$ only for rotational images because $X D S$ has no option to process still images (Rs-0). These custom-made scripts are available on GitHub (https://github.com/keitaroyam/yamtbx).

2.3.4. Sorting of integrated results. Individual integrated results for each image were evaluated based on $\langle I / \sigma(I)\rangle$ values. Note that $\sigma(I)$ values here were derived just from counting statistics for CrystFEL results, whereas for $X D S$ the 
$\sigma(I)$ values estimated by counting statistics were modified by the default error model $\left(a=4, b=10^{-4}\right)$. For the stream file of CrystFEL, we made a script to change the chunk order based on the $\langle I / \sigma(I)\rangle$ values. For $X D S$ results, we sorted the list of filenames that contained the results for each image. The scripts are available on GitHub, as mentioned above.

2.3.5. Anomalous difference Fourier map and SAD phasing. Anomalous difference Fourier peak heights at mercury positions were calculated using ANODE (Thorn \& Sheldrick, 2011). The previously solved cryogenic temperature structure of LRE (PDB code: 5GTQ) was used as the source of phases.

For SAD phasing, the $S H E L X C / D / E$ programs were used (versions 2013/3, 2013/2 and 2016/1, respectively) (Sheldrick, 2010). In SHELXD, 1000 cycles were carried out to search for the heavy atom sites, and the high-resolution cutoff was determined by SHELXC. In SHELXE, 20 cycles of density modification and polyalanine tracing were repeated 60 times. The heavy atom site located by SHELXD was compared with the known site in the refined structure using phenix.emma (Adams et al., 2002) and SHELXE was executed only when the site was correctly located. To determine the minimum required number of images for successful Hg-SAD phasing, the above protocol was first performed for cumulative groups of 1000 images. The lowest number of unsuccessful images was then cumulatively increased by 200 images to find the required minimum.

2.3.6. Structure refinement. The previously solved LRE structure was refined against Ds-1. $1^{i \text { th }}(i=1,2, \ldots, 25)$ to evaluate the occupancies and $B$-values of the mercury atoms. Alternate conformations and waters were removed before refinement. For Ds-1.1 $1^{1 \text { th }}$ and later, only one mercury atom was modeled since the electron density of mercury atoms was so poor. With phenix.refine version 1.10.1 (Afonine et al., 2012), rigid-body refinement followed by refinement of individual atomic coordinates, atomic displacement parameters (ADPs), occupancies of mercury atoms, and automatic water placement were performed. For mercury atoms, anisotropic ADPs were refined and tabulated values of $f^{\prime}$ and $f^{\prime \prime}$ were used. The high-resolution cutoff was $1.6 \AA$. The $m F_{\mathrm{o}}-D F_{\mathrm{c}}$ omit maps were calculated by refining the structure excluding mercury atoms.

Molecular graphics figures were prepared using $P y M O L$ (The PyMOL Molecular Graphics System, Version 1.8, Schrödinger, LLC). All plots were prepared using $R$ (R Development Core Team, 2008) with the ggplot2 package (Wickham, 2009).

\subsection{Estimation of the mosaicity of LRE microcrystals}

The mosaicity of LRE microcrystals was estimated by collecting diffraction data using the conventional rotation method. The beam size and wavelength were the same as those used during SS-ROX data collection. The photon flux was decreased to $0.5 \%$ using a $1500 \mu \mathrm{m}$-thick aluminium absorber. One hundred images were collected from a singlecrystal using a rotation step of $0.1^{\circ}$ and exposure time of $0.1 \mathrm{~s}$.
Data from ten crystals were used to calculate the average mosaicity. The data were processed using XDS (Kabsch, $2010 \mathrm{~b}$ ) and the calculated average mosaicity (estimated standard deviation of the reflection range) was $0.029^{\circ}\left(0.003^{\circ}\right.$ r.m.s.d.).

\section{Results}

\subsection{Data processing}

Datasets Rs-0.1 to Rs-2 were processed using both CrystFEL and XDS, and the results were compared to decide which program was suitable. For processing with $X D S$, we found that discarding reflections with lower partiality and using full intensities estimated by the 3D profiles resulted in higher anomalous signals (Fig. S2 of the supporting information). Generally, partiality estimation is not accurate using a single diffraction image; nonetheless, the full intensity estimation worked better than expected. The optimal partiality cutoff values depended on the rotation width; values of $30 \%$, $50 \%, 70 \%$ and $80 \%$ were obtained for $0.1^{\circ}, 0.25^{\circ}, 0.5^{\circ}$ and $\geq 1.0^{\circ}$, respectively. When processing rotational data using CrystFEL, we applied the Lorentz-factor correction with respect to the goniometer spindle axis. As evaluated using anomalous difference Fourier peak heights as a function of the number of merged images, the Lorentz-factor correction improved the data accuracy, whereas $X D S$ with the optimal partiality cutoff gave a much higher anomalous signal (Fig. S3a). This could be partly because CrystFEL calculates predicted spot positions assuming a still snapshot with finite wavelength dispersion and beam divergence, which results in under-predicted spots and lower multiplicities for larger rotation widths than those determined by $X D S$ (Fig. S3b). In contrast, for smaller rotation widths, multiplicities obtained from CrystFEL were higher than those obtained from $X D S$, but data quality was lower because of the inclusion of lowpartiality reflections that were rejected in the processing using $X D S$. According to these results, we decided to use $X D S$ to process rotational snapshots. However, Rs- 0 was processed using CrystFEL. Datasets Da-1.2 to Da-26 and Ds-1.1 $1^{\text {ith }}(i=$ $1,2, \ldots, 25)$, all of which were collected with a helical rotation step of $0.5^{\circ}$, were processed using $X D S$ with the parameters defined for Rs-0.5.

\subsection{Influence of helical rotation step on SS-ROX}

3.2.1. Hit rate and indexing rate. The hit rates and index rates of Rs-0 to Rs- 2 are presented in Table 1. All hit rates were in the range $36 \%$ to $50 \%$. The low hit rates of Rs- 0 and Rs- 0.1 were caused by the inclusion of one low-hit-rate loop on which the density of microcrystals seemed to be lower compared with that of the other loops. The hit rates calculated by excluding these data were almost the same as those of the other datasets (data not shown), which implies that the hit rate does not depend on the helical rotation step. The indexing rates of Rs- 0.1 to Rs- 2 ranged from $50 \%$ to $62 \%$, and that of Rs- 0 was $81 \%$. 
3.2.2. Resolution limit. The resolution was evaluated using $\mathrm{CC}_{1 / 2}$ and $\langle I / \sigma(I)\rangle$ as criteria for the first 3000 images without sorting (Fig. 2a). A plot of $\mathrm{CC}_{1 / 2}$ against resolution shows that a larger helical rotation step leads to a higher $\mathrm{CC}_{1 / 2}$ up to $2 \AA$. However, the trend changed beyond $2 \AA ; \mathrm{CC}_{1 / 2}$ of Rs-2, Rs-1.5, Rs-1 and Rs-0.5 decreased sharply in this order. When the resolution was cut at $\mathrm{CC}_{1 / 2}=0.5$, the resolution limits increased in the order of Rs-2, Rs- 1.5 , Rs- 1 , Rs- 0.5 and Rs- 0.25 . Rs- 0.25 and Rs- 0.1 had almost the same resolution limit, while Rs-0 had the lowest resolution of all the datasets. These results indicate that decreasing the helical rotation step is effective to improve resolution, but this effect ceases once the helical rotation step is smaller than $0.25^{\circ}$.

Because the exposure time per angle is larger for a smaller rotation step, higher $\mathrm{CC}_{1 / 2}$ and $\langle I / \sigma(I)\rangle$ values were expected for data with a small rotation step. The inverse tendency observed in the low-resolution region might be caused by the higher multiplicity at larger rotation steps. Fig. 2(a) clearly shows that multiplicity increases with rotation step except for Rs-0, which was processed using CrystFEL. To confirm this trend, $\mathrm{CC}_{1 / 2}$ and $\langle I / \sigma(I)\rangle$ were also compared by changing the number of merged images so that all datasets had almost the (a)
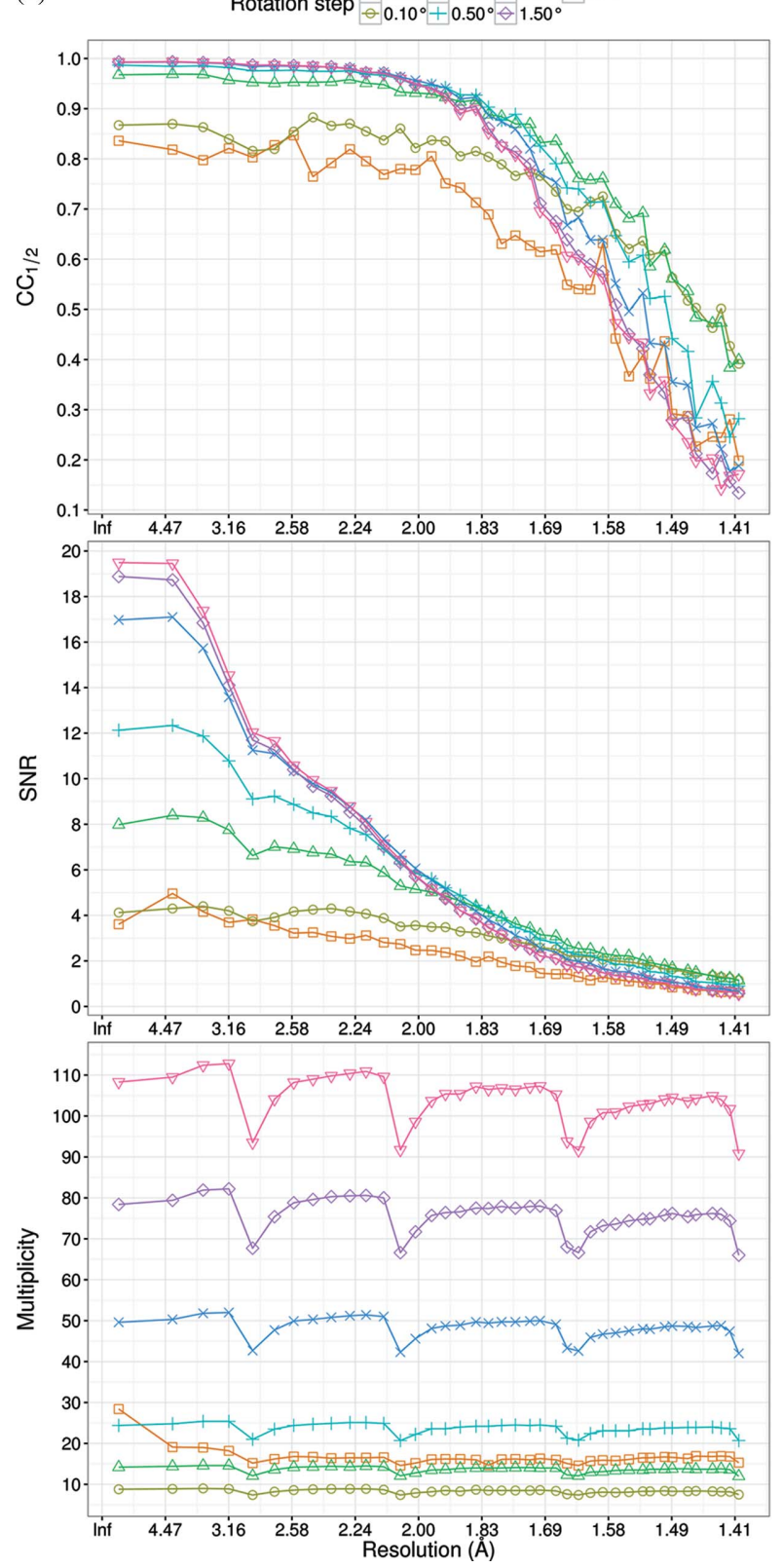

(b)
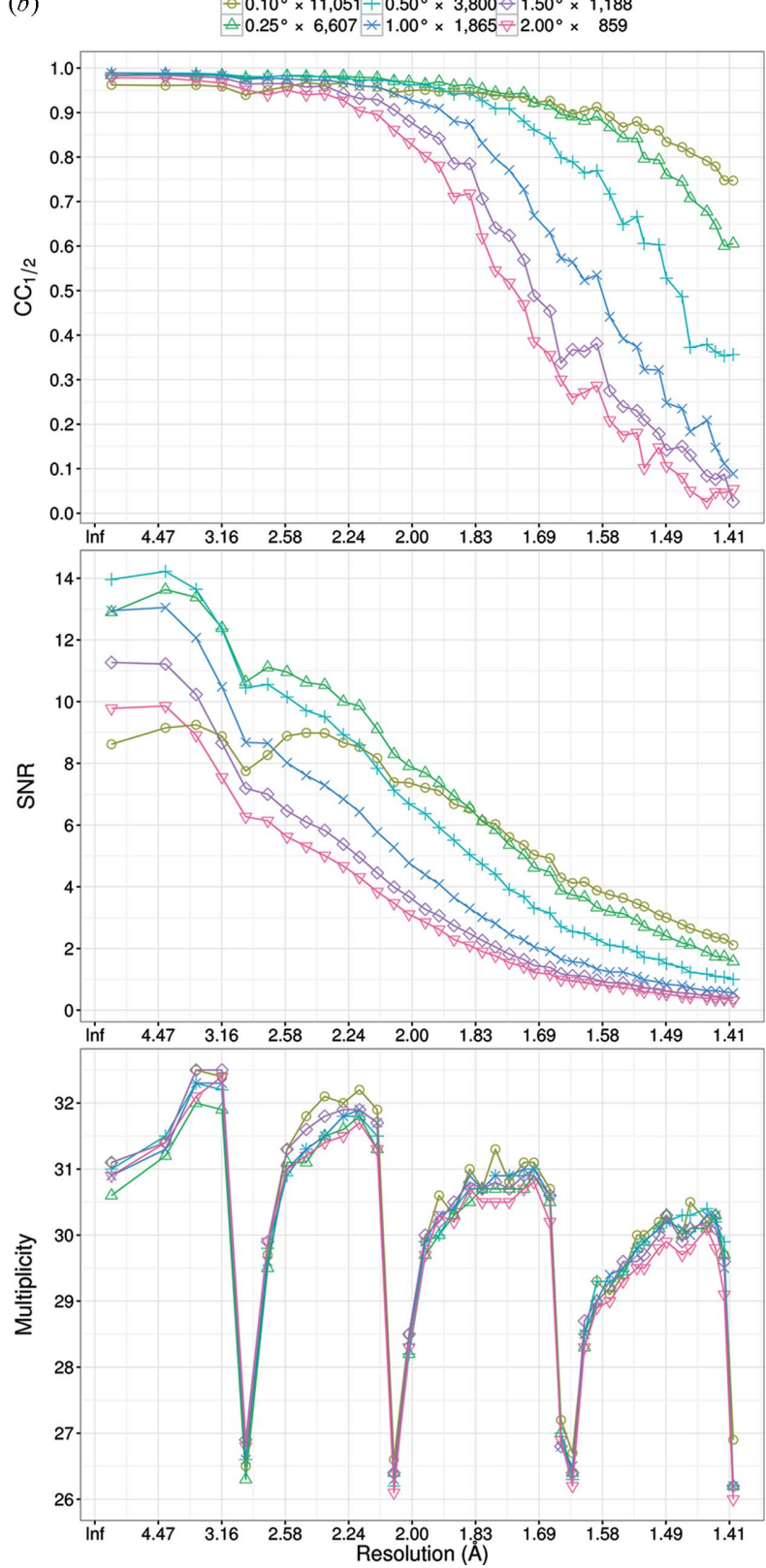

Figure 2

$\mathrm{CC}_{1 / 2},\langle I / \sigma(I)\rangle$ and multiplicity in each resolution shell for Rs-0 to Rs-2.0. (a) 3000 images were merged for each dataset. (b) The number of images was changed so that each dataset had almost the same multiplicity of 30. Decreases of multiplicity at resolutions of 2.9, 2.1, 1.6 and 1.4 Å were caused by dead regions of the PILATUS detector. Images were not sorted on $\langle I / \sigma(I)\rangle$ for these calculations. 
Table 2

Hit rates and indexing rates of datasets collected with various doses.

Photon flux was $2.4 \times 10^{12}$ photons s $^{-1}$ throughout these experiments.

\begin{tabular}{|c|c|c|c|c|c|c|c|c|}
\hline Dataset & $\begin{array}{l}\text { Exposure per } \\
\text { frame (s) }\end{array}$ & $\begin{array}{l}\text { Dose per } \\
\text { frame (MGy) }\end{array}$ & $\begin{array}{l}\text { No. of collected } \\
\text { images }\end{array}$ & $\begin{array}{l}\text { No. of hit } \\
\text { images }\end{array}$ & $\begin{array}{l}\text { No. of indexed } \\
\text { images }\end{array}$ & $\begin{array}{l}\text { Hit rate } \\
(\%)\end{array}$ & $\begin{array}{l}\text { Indexing } \\
\text { rate }(\%)\end{array}$ & $\begin{array}{l}\text { Sample thickness } \\
(\mathrm{mm})\end{array}$ \\
\hline Da-1.2 & 0.02 & 1.2 & 13500 & 6883 & 4546 & 51.0 & 33.7 & 0.13 \\
\hline Da-2.4 & 0.04 & 2.4 & 13500 & 6561 & 4965 & 48.6 & 36.8 & 0.092 \\
\hline Da-4.8 & 0.08 & 4.8 & 13500 & 7575 & 6104 & 56.1 & 45.2 & 0.15 \\
\hline Da-6.7 & 0.111 & 6.7 & 13500 & 7371 & 5872 & 54.6 & 43.5 & 0.12 \\
\hline Da-13 & 0.222 & 13 & 13500 & 7240 & 5903 & 53.6 & 43.7 & 0.12 \\
\hline Da-26 & 0.435 & 26 & 13500 & 8509 & 7696 & 63.0 & 57.0 & 0.16 \\
\hline
\end{tabular}

same multiplicity of 30 (Fig. $2 b$ ). The plots of $\mathrm{CC}_{1 / 2}$ and $\langle I / \sigma(I)\rangle$ against resolution in Fig. 2(b) show that the difference for low-resolution shells was much smaller than that in Fig. 2(a), but Rs-0.1 still had the smallest $\mathrm{CC}_{1 / 2}$ value of all the datasets. This was caused by less accurate estimation of full reflection intensity by $X D S$ because of the smaller partiality. The differences of $\mathrm{CC}_{1 / 2}$ and $\langle I / \sigma(I)\rangle$ for small and large rotation data in Fig. 2(b) were larger than those in Fig. 2(a) at high resolution, which is attributed to the longer total exposure time of small rotation steps than that of large ones because of the larger number of merged images.

3.2.3. Anomalous signal and Hg-SAD phasing. The anomalous signal for Rs-0 to Rs-2 was evaluated by the peak height of the anomalous difference Fourier map as shown in Fig. 3, in which the peak height at the major site is plotted against the number of merged images. For all datasets, the anomalous peak height increased with the number of merged images, demonstrating that an increase in multiplicity improved the accuracy of the anomalous differences. The initial slopes of the curves in Fig. 3 increased with the helical rotation step up to $1^{\circ}$, but then remained almost constant beyond $1^{\circ}$. To estimate

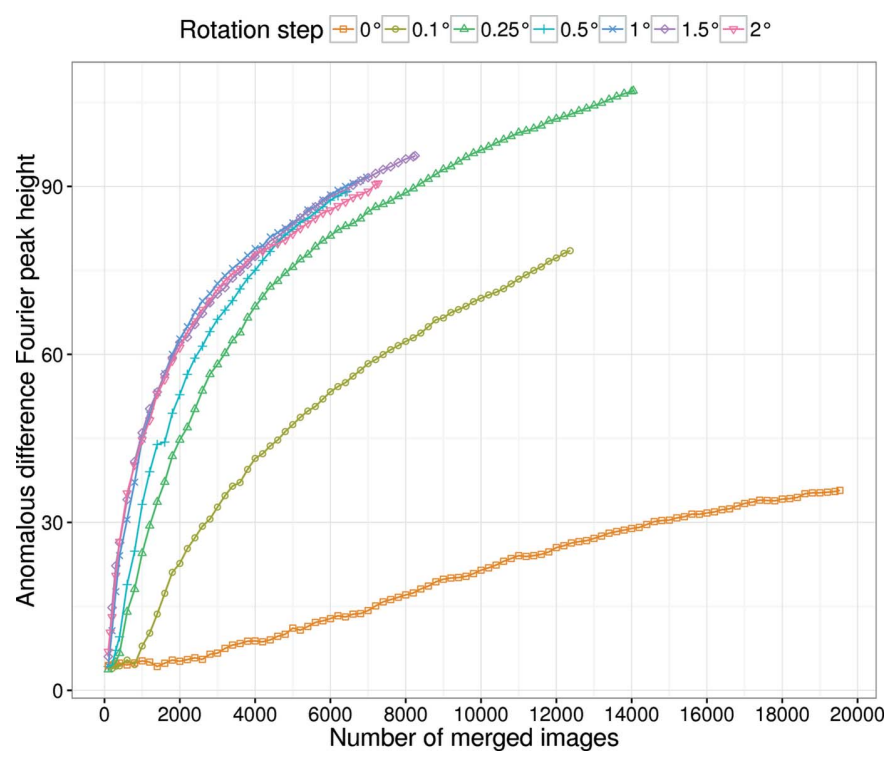

Figure 3

Peak heights of the anomalous difference Fourier map at the Hg site as a function of the number of merged images for Rs-0 to Rs-2. Images were not sorted on $\langle I / \sigma(I)\rangle$ for this calculation. the minimum number of images required for successful $\mathrm{Hg}$ SAD phasing, structure determination was attempted. The minimum number of images was 17800 for Rs-0, 3400 for Rs-0.1, 1200 for Rs- $0.25,1000$ for Rs-0.5, 600 for Rs- 1 and 400 for Rs-1.5 and Rs-2 (Fig. S8a). These results indicate that a helical rotation step of $\geq 1^{\circ}$ is suitable for SAD phasing of LRE.

\subsection{Influence of dose on SS-ROX}

3.3.1. Hit rate and indexing rate. The hit and indexing rates of Da-1.2 to Da-26 are presented in Table 2. The hit rates increased slightly with dose. However, because the variation was comparable with those caused by variation of sample thickness, it was difficult to confirm that the dose contributed to the increase of hit rate.

3.3.2. Resolution limit. Datasets Da-1.2 to Da-26 were collected by increasing the dose from 1.2 to $26 \mathrm{MGy}$ to examine its influence on the data. Figs. $4(a)$ and $4(b)$ display $\mathrm{CC}_{1 / 2}$ and $\langle I / \sigma(I)\rangle$, respectively, of Da-1.2 to Da-26 as a function of resolution. For both $\mathrm{CC}_{1 / 2}$ and $\langle I / \sigma(I)\rangle$, two plots are shown: one was estimated by merging the first 1000 images and the other was estimated by merging the best 1000 images based on $\langle I / \sigma(I)\rangle$ of each image. Comparison of these two plots reveals that the resolution of all datasets is improved by using the best images. Estimating the resolution limit using $\mathrm{CC}_{1 / 2}$ and $\langle I / \sigma(I)\rangle$ derived from the best 1000 images indicated there is a considerable increase in resolution from Da-1.2 to Da-2.4, a significant increase from Da-2.4 to Da-3.4, a marginal increase from Da-3.4 to Da-4.8, a marginal decrease from Da-4.8 to Da-6.7, and a substantial decrease from Da-6.7 to Da-26.

Datasets Ds-1.1 $1^{i \text { th }}(i=1,2, \ldots, 25)$ were collected by repeating the scan 25 times using the same sample with a dose of 1.1 MGy to examine the influence of sample damage on the data. Averaged integrated intensity is plotted against accumulated dose in Fig. S4. Diffraction power decreased to $70 \%$ of its initial value after accumulation of about $10 \mathrm{MGy}$, which is one-third of the Garman limit (Owen et al., 2006). The plots of $\mathrm{CC}_{1 / 2}$ and $\langle I / \sigma(I)\rangle$ against resolution for Ds-1.1 ${ }^{i \text { th }}(i=$ $1,2, \ldots, 25)$ using the best 1000 images in Fig. S5 show a gradual decrease of the resolution limit as the dose accumulated. 
(a)

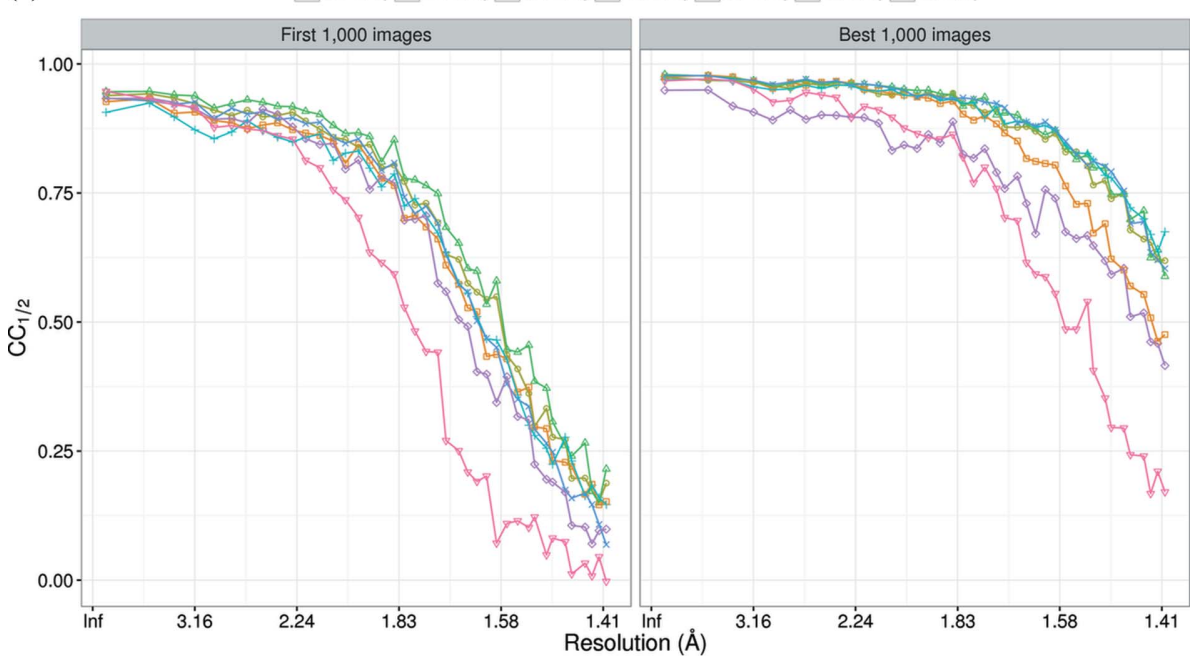

(b)

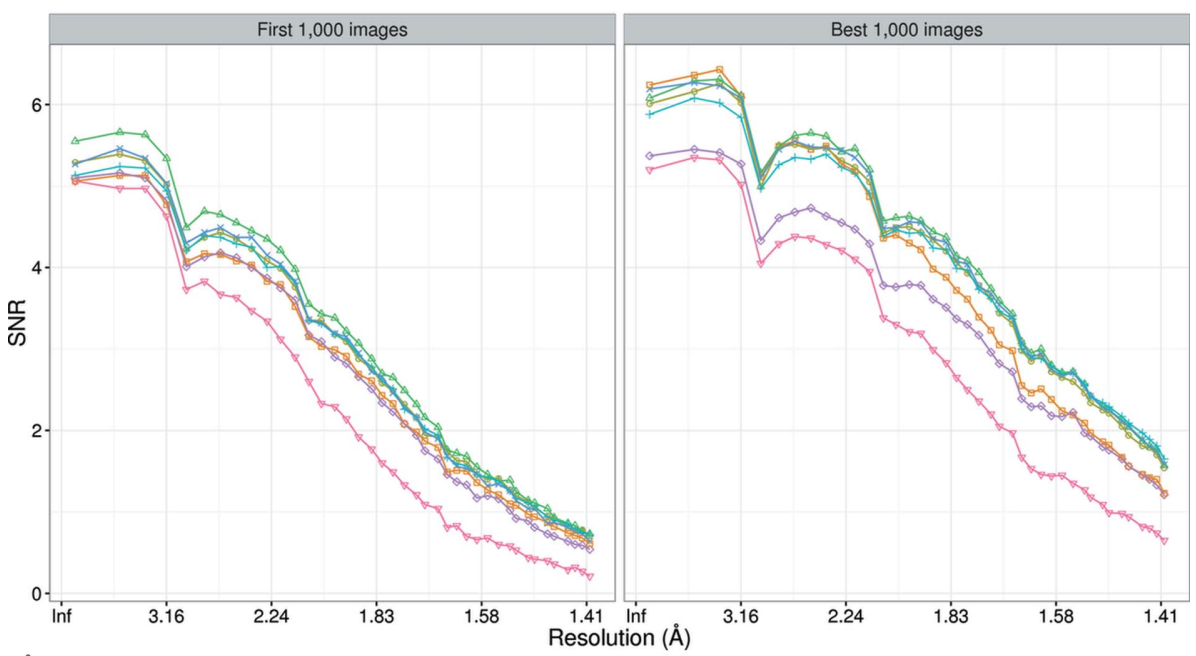

Figure 4

(a) $\mathrm{CC}_{1 / 2}$ and $(b)\langle I / \sigma(I)\rangle$ values for Da-1.2 to Da-26 as a function of resolution. The left panel of each figure was calculated by merging the first 1000 images. The right panel was prepared by merging the best 1000 images, where the best images were selected based on $\langle I / \sigma(I)\rangle$ of each image.

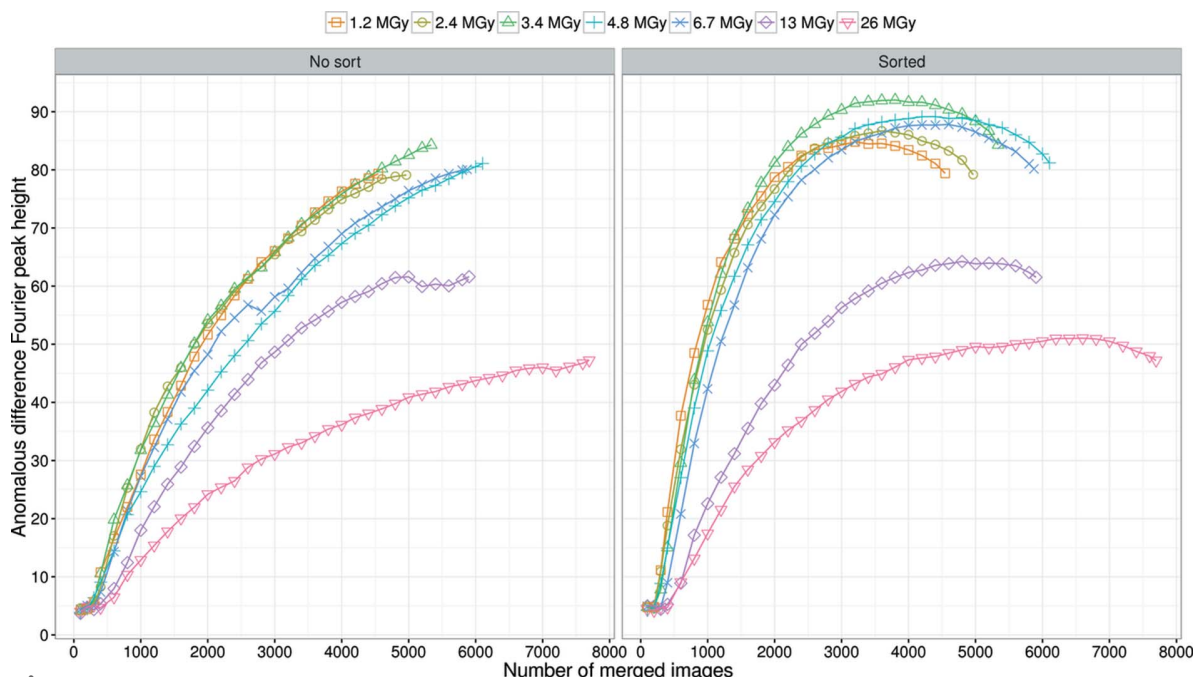

Figure 5

Peak heights of anomalous difference Fourier maps as a function of the number of merged images for Da-1.2 to Da-26. The left figure was prepared without $\langle I / \sigma(I)\rangle$ image sorting. The right figure was prepared after sorting based on $\langle I / \sigma(I)\rangle$ of each image.
3.3.3. Anomalous signal and Hg-SAD phasing. The dose-dependent anomalous signal was evaluated by the peak height of the anomalous difference Fourier map as shown in Fig. 5, where the peak heights of Da-1.2 to Da-26 are plotted against the number of merged images with and without sorting by $\langle I / \sigma(I)\rangle$. Comparison of the plots shown in Fig. 5 demonstrates that sorting made the initial slope of the plots steeper and the peak heights display a convex upward function as the number of merged images increased, indicating that inclusion of lower $\langle I / \sigma(I)\rangle$ images caused the anomalous signal to deteriorate. After sorting, the initial slope of Da-1.2 was the greatest and it decreased with dose. On the other hand, the maximum peak height increased up to $3.4 \mathrm{MGy}$, and started to decrease beyond 3.4 MGy. The maximum peak heights of Da-4.8 and Da-6.7 were comparable with that of Da-3.4, whereas the peak heights of Da-13 and Da-26 were $\leq 75 \%$ of Da-3.4. When the images with highest $\langle I / \sigma(I)\rangle$ values were used, the minimum number of images required for successful $\mathrm{Hg}$-SAD phasing was 600 for Da-1.2, 800 for Da2.4, 600 for $\mathrm{Da}-3.4,1000$ for $\mathrm{Da}-4.8$, 1200 for $\mathrm{Da}-6.7,2400$ for $\mathrm{Da}-13$ and 3200 for Da-26 (Fig. S8b). The corresponding anomalous difference Fourier peak heights are around 50 r.m.s.d. for Da-1.2 to Da-13 and 40 r.m.s.d. for Da-26 (Fig. 5).

The peak heights of the anomalous difference Fourier maps of Ds-1.1 $1^{i \text { th }}(i=$ $1,2, \ldots, 25)$ are presented in Fig. S6. Anomalous peak height gradually decreased with dose accumulation, indicating the existence of radiation damage beyond the absorption of 1.1 MGy. To examine the site-specific damage around the mercury atoms, the structural model was refined for Ds-1.1 $1^{i \text { th }}(i=1,2, \ldots, 25)$. The refined occupancies and atomic $B$-factor values in Fig. 6 showed a gradual decrease in occupancy and increase in $B$-factor together with increasing absorbed dose. The $m F_{\mathrm{o}}-D F_{\mathrm{c}}$ omit maps in Figs. 7 and $\mathrm{S} 7$ show that the electron density around the mercury atoms became more blurred with increasing accumulated dose, which reflects the decrease 


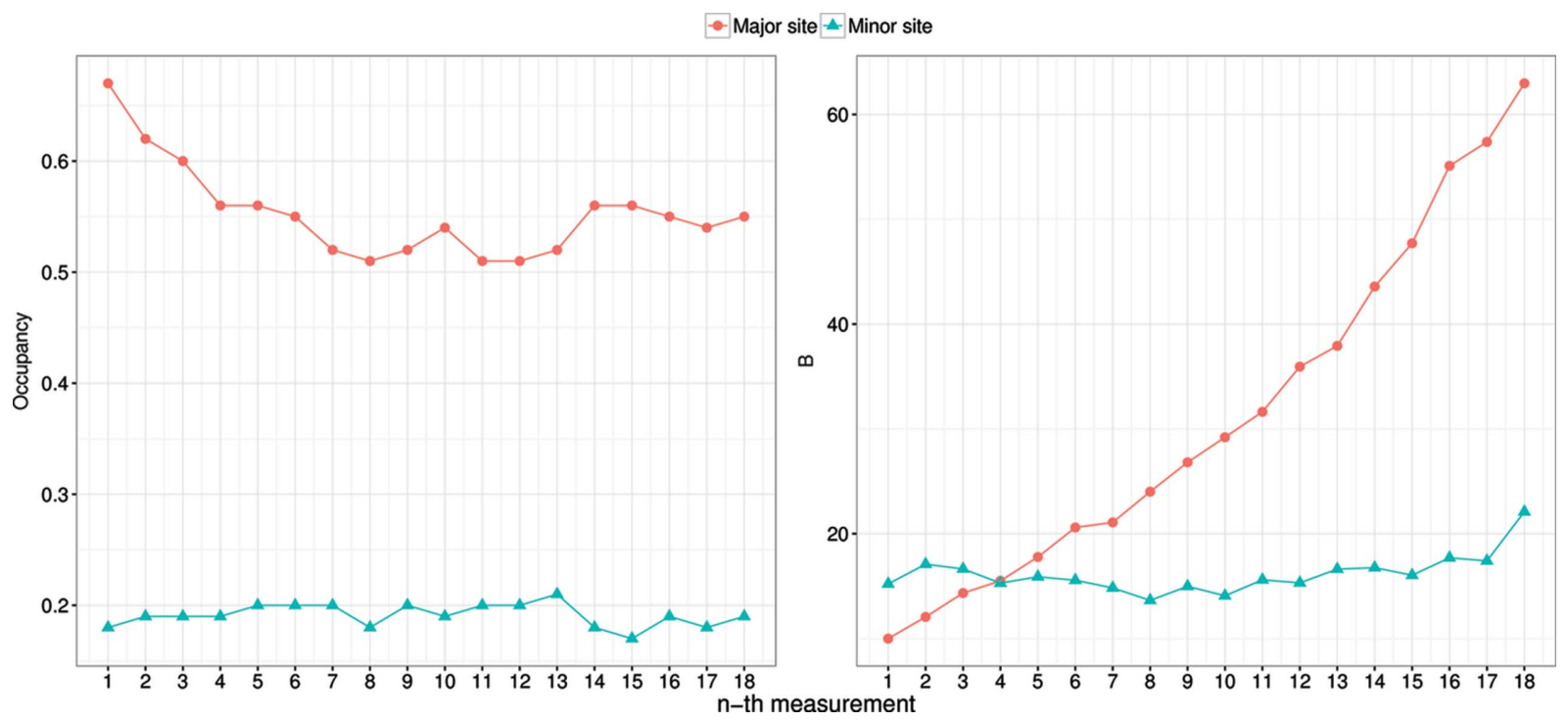

Figure 6

Refined occupancies and $B$ values of Ds-1. $1^{i \text { th }}(i=1,2, \ldots, 18)$. All images were used for each dataset. Red circles are refined values of the major site and blue triangles are those of the minor site. The values for Ds-1.1 $1^{\text {th }}(i=19,20, \ldots, 25)$ were omitted from the plot because they could not be refined properly with the same protocol that was used for the other datasets owing to the low electron density quality of mercury atoms.

in occupancy and increase in $B$-factor value. These data indicate the appearance of specific damage at the mercury site with increasing dose accumulation.

\section{Discussions}

\subsection{Data processing in SS-ROX}

Data collected with goniometer rotation were processed using both CrystFEL and XDS. Our results revealed that $X D S$ outperforms CrystFEL mainly because $X D S$ uses a prediction model for oscillation images and discards low-partiality reflections. Because CrystFEL was developed to process still snapshots, it is not a suitable tool for oscillation data. In contrast, $X D S$ was designed to process a series of continuous frames with increasing spindle angle rotation with a constant step, and estimated the intensity of reflections well using 3D profile fitting. We used only single frames to estimate full intensity by $3 \mathrm{D}$ profile fitting and merged data after applying a single-frame scale factor. Our data showed that use of singleframe 3D profile fitting gave slightly better accuracy than using observed partial intensity if the minimum partiality

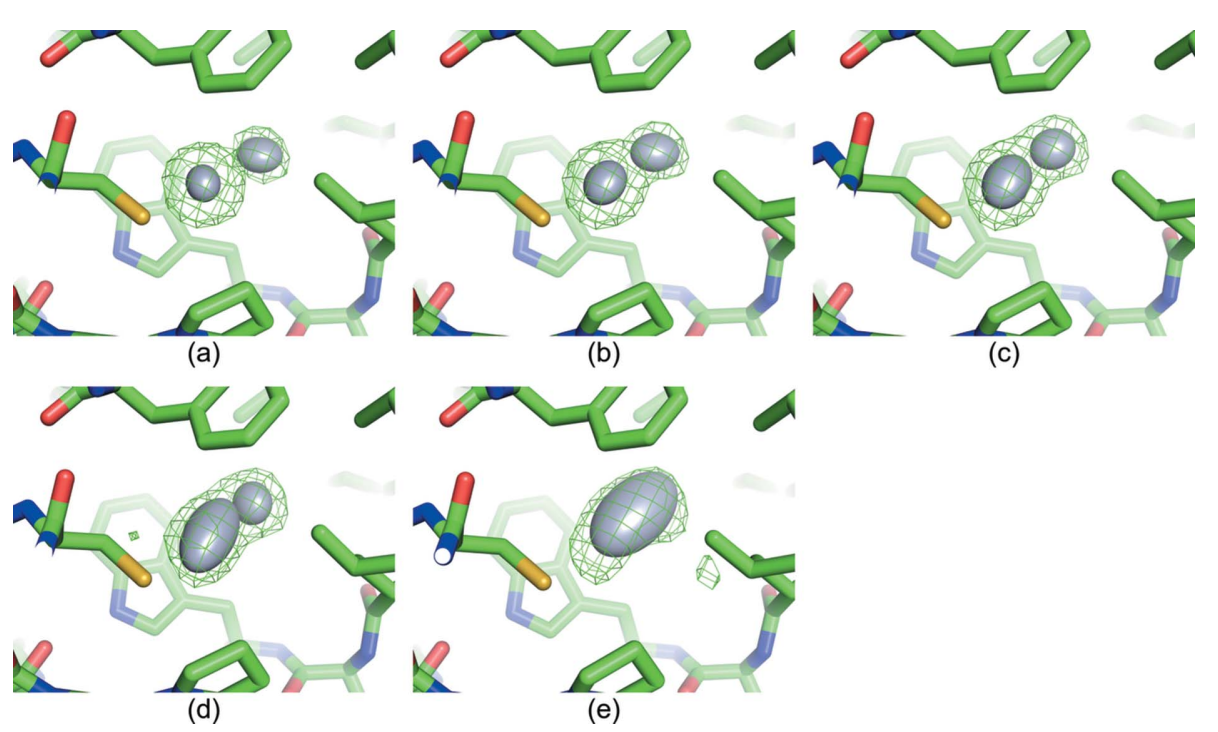

Figure 7

$m F_{\mathrm{o}}-D F_{\mathrm{c}}$ omit maps around the mercury site contoured at 5.0 r.m.s.d. (green mesh). The thermal ellipsoids of mercury atoms are drawn (probability $>0.5$ ) as grey spheroids. Yellow, red, blue and green sticks represent sulfur, oxygen, nitrogen and carbon atoms, respectively. (a) Ds-1.1 $1^{1 \mathrm{st}}$, (b) Ds- $1.1^{3 \mathrm{rd}},(c)$ Ds- $1.1^{\text {th }},(d)$ Ds-1.1 $1^{12 \text { th }}$ and $(e)$ Ds- $1.1^{23 r d}$.
(PEAK) cutoff was selected properly.

A more accurate full-intensity estimation can be expected if a postrefinement program is used, $n X D S$ (Kabsch, 2014) being one candidate. However, there is another difficulty in processing SS-ROX data; the diffraction image can be 'incomplete', meaning that the effective rotation step of a crystal can be smaller than that of the goniometer. This is because continuous translation during the helical scan can result in the crystal moving in and/ or out of the incident X-ray beam. This incomplete rotation complicates post refinement, but an improvement in data accuracy can be expected if the data are treated properly. The possibility of incomplete rotation depends on the helical translation step as well as both crystal and beam size. Here a beam size comparable with the smallest dimension of the crystals was used, and all data 


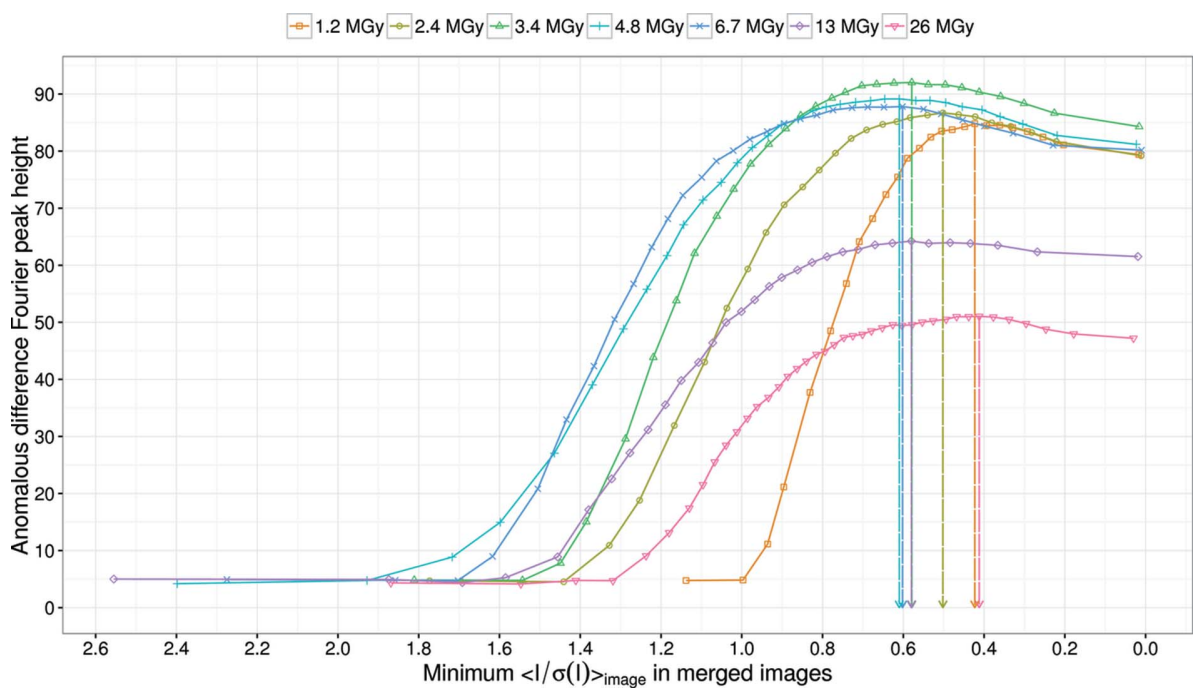

Figure 8

Peak heights of the anomalous difference Fourier maps of Da-1.2 to Da-26 as a function of the rejection criteria of the $\langle I / \sigma(I)\rangle$ value. Vertical arrows indicate the $\langle I / \sigma(I)\rangle$ values that give the best results. Maximum peak height was observed in the $\langle I / \sigma(I)\rangle$ range from 0.4 to 0.6.

reflection is recorded over several consecutive frames, which has the advantage of decreasing background scattering. Although this method has suffered from jitter caused by inadequate shutter and goniometer control and long data collection times originating from slow detector readout, shutterless data collection using a fastreadout detector, such as a pixel array or CMOS detector, has solved these problems and a fine rotation step is now widely used. However, it has been pointed out that a smaller rotation step does not necessarily give good results. Hasegawa et al. (2009) showed that a rotation step that was too small was not beneficial because of the readout noise of the CMOS detector which was used. Mueller et al. (2012) proposed that the optimum rotation step for a PILATUS detector was half the crystal mosaicity

were collected using a translation step equal to the horizontal beam size. If a smaller helical translation step is used, the possibility of incomplete rotation decreases and more accurate estimation of the diffraction intensity might be obtained.

While processing Da-1.2 to Da-26, we noticed the importance of sorting and rejecting low-quality images prior to merging. In serial crystallography data collection, there may be variation in crystal size and quality, and there is also the possibility that only a small region of the crystal is exposed to X-ray beam. All of these factors result in variation of $S / N$. Inclusion of images with low $\mathrm{S} / \mathrm{N}$ deteriorates the achievable resolution, as shown in Figs. 4(a) and 4(b), or results in the deterioration of the anomalous signal (Fig. 5). Therefore, rejection of lower quality data is necessary to improve the data accuracy. Here, using the $\langle I / \sigma(I)\rangle$ of individual images as a sorting key worked well. Our study also gave some insights into the threshold of rejection to maximize anomalous signal. Fig. 8 shows the peak height of the anomalous difference Fourier maps of Da-1.2 to Da-26 as a function of the rejection criteria of $\langle I / \sigma(I)\rangle$. A threshold of 0.4 to 0.6 maximizes the anomalous signal.

\subsection{Influence of the helical rotation step on SS-ROX}

Our results demonstrated that rotation was effective in SSX. In the case of LRE microcrystals, the best rotation step to achieve the highest resolution was $0.25^{\circ}$ and a helical rotation step larger than or equal to $1^{\circ}$ was better for $\mathrm{Hg}$-SAD phasing. Both rotational steps were larger than the mosaicity of LRE microcrystals estimated by XDS $\left(0.03^{\circ}\right)$.

The advantages of using a fine rotation step for conventional rotation data collection methods has been examined in several studies (Pflugrath, 1999; Hülsen et al., 2006; Hasegawa et al., 2009; Mueller et al., 2012; Casanas et al., 2016). When a rotation step smaller than the reflecting range is used, one estimated by $X D S$. Further decreasing the rotation step could reduce the accuracy because of the dead-time of the detector and/or the poor estimation of intensity from the integration software. Recently, Casanas et al. (2016) reported that the decrease of the rotation step down to one-tenth of mosaicity estimated by $X D S$ was effective for data collection using the Eiger detector.

These differences between SS-ROX and conventional oscillation methods could be attributed to the difference of data collection and processing methods used in SS-ROX; one crystal or one region of a crystal gives only one diffraction image and the data are merged by Monte Carlo integration instead of conventional 3D profile fitting using consecutive rotational images. Therefore, in SS-ROX, the full intensity is not necessarily integrated from adjacent frames. The advantage of a larger helical rotation step in SS-ROX is wider coverage of reciprocal space, which contributes to higher multiplicity. It also contributes to recording diffraction spots as full reflections. Even when recorded as partial reflections, higher partiality is advantageous for accurate estimation of full reflection intensities by $X D S$. Conversely, a smaller rotation step is expected to have a higher $\mathrm{S} / \mathrm{N}$ caused by the longer exposure time per rotation angle as well as lower background scattering. The effect of background scattering caused by sample thickness is also expected to be small when the helical rotation step is small, while large rotation increases the beam path on the sample and leads to larger background scattering. Therefore, the optimal helical rotation step in SS-ROX might be determined by a combination of these factors. Our data show that the dominant factor for determining the optimal helical rotation step differs depending on the resolution range; higher multiplicity and larger partiality were advantageous for low resolution, whereas longer exposure time per rotation angle and/or lower background scattering made important contributions at high resolution. For $\mathrm{Hg}-\mathrm{SAD}$ phasing, a 
rotation step $\geq 1^{\circ}$ was favorable in the case of LRE microcrystals. We speculate that this was because the anomalous signal was dominated by low-resolution reflections, where a larger rotation step gives higher accuracy.

As shown in Fig. S2, discarding low-partiality reflections was important. In our case, where mosaicity was $0.03^{\circ}$, most reflections had low partialities with $0.1^{\circ}$ rotation, so larger rotations were beneficial to lower the fraction of low-partiality reflections. For other cases with higher mosaicity, optimal rotation could be larger and spot overlaps could be a problem. Further study using several samples is underway.

\subsection{Influence of dose on SS-ROX}

The resolution limit of SS-ROX is assumed to be determined by the balance between the counting statistics and radiation damage. A longer exposure time decreases the counting error, but signal deterioration caused by radiation damage may occur when the exposure time is long. Even though a gradual decrease of resolution was observed beyond absorption of $1.1 \mathrm{MGy}$ (Fig. S5), achievable resolution increased up to $3.4 \mathrm{MGy}$ (Fig. 4) in the case of LRE microcrystals. This shows that a longer exposure time improves the $\mathrm{S} / \mathrm{N}$ of weak reflections for high-resolution shells despite the existence of radiation damage.

The anomalous peak height after sorting in Fig. 5 revealed that the initial slope of the Da-1.2 plot was the steepest and gradually decreased with increasing dose. However, Da-3.4 displays the maximum peak height among the seven datasets, which was attributed to the larger $\mathrm{S} / \mathrm{N}$ caused by the longer exposure time because the difference of number of merged images at the maximum peak height was small for Da-1.2, Da-2.4 and Da-3.4 (Fig. 5). Conversely, the maximum anomalous peak height began to decrease gradually beyond 3.4 MGy, implying that there was no merit in going beyond 3.4 MGy in the case of the mercury derivative of LRE. This limit will change for different heavy atom derivatives and/or proteins because the susceptibility of heavy atoms to radiation damage depends on both the type of heavy atom and its surrounding environment (Ravelli et al., 2005; Ramagopal et al., 2005). The deterioration of the anomalous signal at higher dose could be attributed to site-specific damage. Comparison of Ds-1.1 $1^{i \text { th }}(i=1,2, \ldots, 25)$ showed the propagation of sitespecific damage at the mercury site (Fig. 7). Such disordering and/or partial disruption of mercury could change the relative intensity of reflections and lead to less accurate estimation of the Bijvoet differences (Owen \& Sherrell, 2016).

Because the LRE microcrystals were of good quality with diffraction beyond $1.6 \AA$, we succeeded in SAD phasing using an exposure time of $0.02 \mathrm{~s}$, which corresponds to a dose of 1.2 MGy. However, when the diffraction power was insufficient, the dose limit demonstrated here became critical; hence, data collection strategy must be considered carefully. One approach is to increase the multiplicity by using multiple samples, based on the same concept as multicrystal data collection.

\section{Conclusion}

We have demonstrated the advantages and limitations of SS-ROX by evaluating the anomalous signal and $\mathrm{Hg}$-SAD phasing of LRE. An exposure time of milliseconds to seconds at synchrotron beamlines allowed rotation during the X-ray exposure, which enabled complete data with high accuracy to be collected efficiently. Our data show that the optimum helical rotation step in SS-ROX can be determined depending on various factors such as the multiplicity and the partiality of reflections, exposure time per rotation angle and the contribution from background scattering. For the LRE microcrystals, $0.25^{\circ}$ was the best rotation step for the achievable resolution limit, whereas a rotation larger than or equal to $1^{\circ}$ was better for Hg-SAD phasing, where the contribution of low-resolution reflections is important. Radiation damage limited the exposure time in SS-ROX. Specific damage affecting the $\mathrm{Hg}$ site appeared for accumulated doses beyond 1.1 MGy, but increases in resolution and anomalous signal were observed up to $3.4 \mathrm{MGy}$ because of the higher S/N of data. This limitation could be mitigated by using multiple samples. Although limited by radiation damage, we conclude that efficient data collection by SS-ROX will make it a powerful tool for protein microcrystallography.

\section{Acknowledgements}

The experiments at SPring-8 BL41XU were carried out with the approval of the Japan Synchrotron Radiation Research Institute (JASRI; proposal Nos. 2014A1001, 2014A1464, 2014A1900, 2014B1002, 2014B1450, 2014B2058, 2015A1001, 2015A1094, 2015A2063, 2015B1035, 2015B1650, 2015B2005, 2015B2094, 2016A1001, 2016A1856 and 2016A2735). This work was partly supported by the Platform for Drug Discovery, Informatics and Structural Life Science (MEXT). The authors thank H. Yumoto, Y. Senba and H. Ohashi at JASRI for their help with the installation of the new focusing optics at BL41XU to enable microcrystallography. We acknowledge Y. Furukawa at JASRI for his help with the setup of a control system for the raster scan. We thank T. Halsted and T. Nakane for careful reading of the manuscript, and K. Ohmichi and Y. Ohtsuka for their help with sample preparation. Raw diffraction data have been deposited at CXIDB (http://cxidb.org/) with CXIDB ID 46. Atomic coordinates of LRE models refined against Ds-1.1 ${ }^{1 \mathrm{st}}$, Ds-1.1 ${ }^{3 \mathrm{rd}}$, Ds- $1.1^{\text {th }}$, Ds- $1.1^{12 \text { th }}$ and Ds- $1.1^{23 r d}$ are deposited in the PDB with accession codes 5GX1, 5GX2, 5GX3, 5GX4 and 5GX5, respectively.

\section{References}

Adams, P. D., Grosse-Kunstleve, R. W., Hung, L.-W., Ioerger, T. R., McCoy, A. J., Moriarty, N. W., Read, R. J., Sacchettini, J. C., Sauter, N. K. \& Terwilliger, T. C. (2002). Acta Cryst. D58, 1948-1954.

Afonine, P. V., Grosse-Kunstleve, R. W., Echols, N., Headd, J. J., Moriarty, N. W., Mustyakimov, M., Terwilliger, T. C., Urzhumtsev, A., Zwart, P. H. \& Adams, P. D. (2012). Acta Cryst. D68, 352-367.

Barends, T. R. M., Foucar, L., Botha, S., Doak, R. B., Shoeman, R. L., Nass, K., Koglin, J. E., Williams, G. J., Boutet, S., Messerschmidt, M. \& Schlichting, I. (2014). Nature (London), 505, 244-247. 
Barty, A., Kirian, R. A., Maia, F. R. N. C., Hantke, M., Yoon, C. H., White, T. A. \& Chapman, H. (2014). J. Appl. Cryst. 47, 1118-1131. Botha, S., Nass, K., Barends, T. R. M., Kabsch, W., Latz, B., Dworkowski, F., Foucar, L., Panepucci, E., Wang, M., Shoeman, R. L., Schlichting, I. \& Doak, R. B. (2015). Acta Cryst. D71, 387397.

Boutet, S., Lomb, L., Williams, G. J., Barends, T. R. M., Aquila, A., Doak, R. B., Weierstall, U., DePonte, D. P., Steinbrener, J., Shoeman, R. L., Messerschmidt, M., Barty, A., White, T. A., Kassemeyer, S., Kirian, R. A., Seibert, M. M., Montanez, P. A., Kenney, C., Herbst, R., Hart, P., Pines, J., Haller, G., Gruner, S. M., Philipp, H. T., Tate, M. W., Hromalik, M., Koerner, L. J., van Bakel, N., Morse, J., Ghonsalves, W., Arnlund, D., Bogan, M. J., Caleman, C., Fromme, R., Hampton, C. Y., Hunter, M. S., Johansson, L., Katona, G., Kupitz, C., Liang, M., Martin, A. V., Nass, K., Redecke, L., Stellato, F., Timneanu, N., Wang, D., Zatsepin, N. A., Schafer, D., Defever, J., Neutze, R., Fromme, P., Spence, J. C. H., Chapman, H. N. \& Schlichting, I. (2012). Science, 337, 362-364.

Burmeister, W. P. (2000). Acta Cryst. D56, 328-341.

Casanas, A., Warshamanage, R., Finke, A. D., Panepucci, E., Olieric, V., Nöll, A., Tampé, R., Brandstetter, S., Förster, A., Mueller, M., Schulze-Briese, C., Bunk, O. \& Wang, M. (2016). Acta Cryst. D72, 1036-1048.

Chapman, H. N., Fromme, P., Barty, A., White, T. A., Kirian, R. A., Aquila, A., Hunter, M. S., Schulz, J., DePonte, D. P., Weierstall, U., Doak, R. B., Maia, F. R. N. C., Martin, A. V., Schlichting, I., Lomb, L., Coppola, N., Shoeman, R. L., Epp, S. W., Hartmann, R., Rolles, D., Rudenko, A., Foucar, L., Kimmel, N., Weidenspointner, G., Holl, P., Liang, M., Barthelmess, M., Caleman, C., Boutet, S., Bogan, M. J., Krzywinski, J., Bostedt, C., Bajt, S., Gumprecht, L., Rudek, B., Erk, B., Schmidt, C., Hömke, A., Reich, C., Pietschner, D., Strüder, L., Hauser, G., Gorke, H., Ullrich, J., Herrmann, S., Schaller, G., Schopper, F., Soltau, H., Kühnel, K.-U., Messerschmidt, M., Bozek, J. D., Hau-Riege, S. P., Frank, M., Hampton, C. Y., Sierra, R. G., Starodub, D., Williams, G. J., Hajdu, J., Timneanu, N., Seibert, M. M., Andreasson, J., Rocker, A., Jönsson, O., Svenda, M., Stern, S., Nass, K., Andritschke, R., Schröter, C.-D., Krasniqi, F., Bott, M., Schmidt, K. E., Wang, X., Grotjohann, I., Holton, J. M., Barends, T. R. M., Neutze, R., Marchesini, S., Fromme, R., Schorb, S., Rupp, D., Adolph, M., Gorkhover, T., Andersson, I., Hirsemann, H., Potdevin, G., Graafsma, H., Nilsson, B. \& Spence, J. C. H. (2011). Nature (London), 470, 73-77.

Coquelle, N., Brewster, A. S., Kapp, U., Shilova, A., Weinhausen, B., Burghammer, M. \& Colletier, J.-P. (2015). Acta Cryst. D71, 11841196.

Duisenberg, A. J. M. (1992). J. Appl. Cryst. 25, 92-96.

Fischetti, R. F., Xu, S., Yoder, D. W., Becker, M., Nagarajan, V., Sanishvili, R., Hilgart, M. C., Stepanov, S., Makarov, O. \& Smith, J. L. (2009). J. Synchrotron Rad. 16, 217-225.

Flot, D., Mairs, T., Giraud, T., Guijarro, M., Lesourd, M., Rey, V., van Brussel, D., Morawe, C., Borel, C., Hignette, O., Chavanne, J., Nurizzo, D., McSweeney, S. \& Mitchell, E. (2010). J. Synchrotron Rad. 17, 107-118.

Fukuda, Y., Tse, K. M., Nakane, T., Nakatsu, T., Suzuki, M., Sugahara, M., Inoue, S., Masuda, T., Yumoto, F., Matsugaki, N., Nango, E., Tono, K., Joti, Y., Kameshima, T., Song, C., Hatsui, T., Yabashi, M., Nureki, O., Murphy, M. E. P., Inoue, T., Iwata, S. \& Mizohata, E. (2016). Proc. Natl Acad. Sci. USA, 113, 2928-2933.

Garman, E. F. (2010). Acta Cryst. D66, 339-351.

Gati, C., Bourenkov, G., Klinge, M., Rehders, D., Stellato, F., Oberthür, D., Yefanov, O., Sommer, B. P., Mogk, S., Duszenko, M., Betzel, C., Schneider, T. R., Chapman, H. N. \& Redecke, L. (2014). IUCrJ, 1, 87-94.

González, A. (2007). J. Synchrotron Rad. 14, 43-50.

Hasegawa, K., Hirata, K., Shimizu, T., Shimizu, N., Hikima, T., Baba, S., Kumasaka, T. \& Yamamoto, M. (2009). J. Appl. Cryst. 42, 11651175.

Henderson, R. (1990). Proc. R. Soc. London B, 241, 6-8.
Hirata, K., Kawano, Y., Ueno, G., Hashimoto, K., Murakami, H., Hasegawa, K., Hikima, T., Kumasaka, T. \& Yamamoto, M. (2013). J. Phys. Conf. Ser. 425, 012002.

Hirata, K. et al. (2017). In preparation.

Holton, J. M. (2009). J. Synchrotron Rad. 16, 133-142.

Huang, C.-Y., Olieric, V., Ma, P., Howe, N., Vogeley, L., Liu, X., Warshamanage, R., Weinert, T., Panepucci, E., Kobilka, B., Diederichs, K., Wang, M. \& Caffrey, M. (2016). Acta Cryst. D72, 93-112.

Huang, C.-Y., Olieric, V., Ma, P., Panepucci, E., Diederichs, K., Wang, M. \& Caffrey, M. (2015). Acta Cryst. D71, 1238-1256.

Hülsen, G., Broennimann, C., Eikenberry, E. F. \& Wagner, A. (2006). J. Appl. Cryst. 39, 550-557.

Kabsch, W. (2010a). Acta Cryst. D66, 133-144.

Kabsch, W. (2010b). Acta Cryst. D66, 125-132.

Kabsch, W. (2014). Acta Cryst. D70, 2204-2216.

Kern, J., Tran, R., Alonso-Mori, R., Koroidov, S., Echols, N., Hattne, J., Ibrahim, M., Gul, S., Laksmono, H., Sierra, R. G., Gildea, R. J., Han, G., Hellmich, J., Lassalle-Kaiser, B., Chatterjee, R., Brewster, A. S., Stan, C. A., Glöckner, C., Lampe, A., DiFiore, D., Milathianaki, D., Fry, A. R., Seibert, M. M., Koglin, J. E., Gallo, E., Uhlig, J., Sokaras, D., Weng, T.-C., Zwart, P. H., Skinner, D. E., Bogan, M. J., Messerschmidt, M., Glatzel, P., Williams, G. J., Boutet, S., Adams, P. D., Zouni, A., Messinger, J., Sauter, N. K., Bergmann, U., Yano, J. \& Yachandra, V. K. (2014). Nat. Commun. 5, 4371.

Kupitz, C., Basu, S., Grotjohann, I., Fromme, R., Zatsepin, N. A., Rendek, K. N., Hunter, M. S., Shoeman, R. L., White, T. A., Wang, D., James, D., Yang, J.-H., Cobb, D. E., Reeder, B., Sierra, R. G., Liu, H., Barty, A., Aquila, A. L., Deponte, D., Kirian, R. A., Bari, S., Bergkamp, J. J., Beyerlein, K. R., Bogan, M. J., Caleman, C., Chao, T.-C., Conrad, C. E., Davis, K. M., Fleckenstein, H., Galli, L., Hau-Riege, S. P., Kassemeyer, S., Laksmono, H., Liang, M., Lomb, L., Marchesini, S., Martin, A. V., Messerschmidt, M., Milathianaki, D., Nass, K., Ros, A., Roy-Chowdhury, S., Schmidt, K., Seibert, M., Steinbrener, J., Stellato, F., Yan, L., Yoon, C., Moore, T. A., Moore, A. L., Pushkar, Y., Williams, G. J., Boutet, S., Doak, R. B., Weierstall, U., Frank, M., Chapman, H. N., Spence, J. C. H. \& Fromme, P. (2014). Nature (London), 513, 261-265.

Liebschner, D., Rosenbaum, G., Dauter, M. \& Dauter, Z. (2015). Acta Cryst. D71, 772-778.

Liu, W., Wacker, D., Gati, C., Han, G. W., James, D., Wang, D., Nelson, G., Weierstall, U., Katritch, V., Barty, A., Zatsepin, N. A., Li, D., Messerschmidt, M., Boutet, S., Williams, G. J., Koglin, J. E., Seibert, M. M., Wang, C., Shah, S. T. A., Basu, S., Fromme, R., Kupitz, C., Rendek, K. N., Grotjohann, I., Fromme, P., Kirian, R. A., Beyerlein, K. R., White, T. A., Chapman, H. N., Caffrey, M., Spence, J. C. H., Stevens, R. C. \& Cherezov, V. (2013). Science, 342, 1521-1524.

Mueller, M., Wang, M. \& Schulze-Briese, C. (2012). Acta Cryst. D68, $42-56$.

Nakane, T., Song, C., Suzuki, M., Nango, E., Kobayashi, J., Masuda, T., Inoue, S., Mizohata, E., Nakatsu, T., Tanaka, T., Tanaka, R., Shimamura, T., Tono, K., Joti, Y., Kameshima, T., Hatsui, T., Yabashi, M., Nureki, O., Iwata, S. \& Sugahara, M. (2015). Acta Cryst. D71, 2519-2525.

Nass, K., Meinhart, A., Barends, T. R. M., Foucar, L., Gorel, A., Aquila, A., Botha, S., Doak, R. B., Koglin, J., Liang, M., Shoeman, R. L., Williams, G., Boutet, S. \& Schlichting, I. (2016). IUCrJ, 3, 180-191.

Neutze, R., Wouts, R., van der Spoel, D., Weckert, E. \& Hajdu, J. (2000). Nature (London), 406, 752-757.

Nogly, P., James, D., Wang, D., White, T. A., Zatsepin, N., Shilova, A., Nelson, G., Liu, H., Johansson, L., Heymann, M., Jaeger, K., Metz, M., Wickstrand, C., Wu, W., Båth, P., Berntsen, P., Oberthuer, D., Panneels, V., Cherezov, V., Chapman, H., Schertler, G., Neutze, R., Spence, J., Moraes, I., Burghammer, M., Standfuss, J. \& Weierstall, U. (2015). IUCrJ, 2, 168-176.

Owen, R. L., Rudiño-Piñera, E. \& Garman, E. F. (2006). Proc. Natl Acad. Sci. USA, 103, 4912-4917. 
Owen, R. L. \& Sherrell, D. A. (2016). Acta Cryst. D72, 388-394.

Pflugrath, J. W. (1999). Acta Cryst. D55, 1718-1725.

Ramagopal, U. A., Dauter, Z., Thirumuruhan, R., Fedorov, E. \& Almo, S. C. (2005). Acta Cryst. D61, 1289-1298.

Ravelli, R. B. \& McSweeney, S. M. (2000). Structure, 8, 315-328.

Ravelli, R. B. G., Nanao, M. H., Lovering, A., White, S. \& McSweeney, S. (2005). J. Synchrotron Rad. 12, 276-284.

R Development Core Team (2008). R: A Language and Environment for Statistical Computing. R Foundation for Statistical Computing, Vienna, Austria.

Redecke, L., Nass, K., DePonte, D. P., White, T. A., Rehders, D., Barty, A., Stellato, F., Liang, M., Barends, T. R., Boutet, S., Williams, G. J., Messerschmidt, M., Seibert, M. M., Aquila, A., Arnlund, D., Bajt, S., Barth, T., Bogan, M. J., Caleman, C., Chao, T.-C., Doak, R. B., Fleckenstein, H., Frank, M., Fromme, R., Galli, L., Grotjohann, I., Hunter, M. S., Johansson, L. C., Kassemeyer, S., Katona, G., Kirian, R. A., Koopmann, R., Kupitz, C., Lomb, L., Martin, A. V., Mogk, S., Neutze, R., Shoeman, R. L., Steinbrener, J., Timneanu, N., Wang, D., Weierstall, U., Zatsepin, N. A., Spence, J. C., Fromme, P., Schlichting, I., Duszenko, M., Betzel, C. \& Chapman, H. N. (2013). Science, 339, 227-230.

Riekel, C., Burghammer, M. \& Schertler, G. (2005). Curr. Opin. Struct. Biol. 15, 556-562.

Schiltz, M., Dumas, P., Ennifar, E., Flensburg, C., Paciorek, W., Vonrhein, C. \& Bricogne, G. (2004). Acta Cryst. D60, 1024-1031.

Sheldrick, G. M. (2010). Acta Cryst. D66, 479-485.

Smith, J. L., Fischetti, R. F. \& Yamamoto, M. (2012). Curr. Opin. Struct. Biol. 22, 602-612.

Stellato, F., Oberthür, D., Liang, M., Bean, R., Gati, C., Yefanov, O., Barty, A., Burkhardt, A., Fischer, P., Galli, L., Kirian, R. A., Meyer, J., Panneerselvam, S., Yoon, C. H., Chervinskii, F., Speller, E., White, T. A., Betzel, C., Meents, A. \& Chapman, H. N. (2014). IUCrJ, 1, 204-212.

Tenboer, J., Basu, S., Zatsepin, N., Pande, K., Milathianaki, D., Frank, M., Hunter, M., Boutet, S., Williams, G. J., Koglin, J. E., Oberthuer, D., Heymann, M., Kupitz, C., Conrad, C., Coe, J., Roy-Chowdhury, S., Weierstall, U., James, D., Wang, D., Grant, T., Barty, A., Yefanov,
O., Scales, J., Gati, C., Seuring, C., Srajer, V., Henning, R., Schwander, P., Fromme, R., Ourmazd, A., Moffat, K., Van Thor, J. J., Spence, J. C. H., Fromme, P., Chapman, H. N. \& Schmidt, M. (2014). Science, 346, 1242-1246.

Thorn, A. \& Sheldrick, G. M. (2011). J. Appl. Cryst. 44, 12851287.

Weierstall, U., James, D., Wang, C., White, T. A., Wang, D., Liu, W., Spence, J. C. H., Bruce Doak, R., Nelson, G., Fromme, P., Fromme, R., Grotjohann, I., Kupitz, C., Zatsepin, N. A., Liu, H., Basu, S., Wacker, D., Won Han, G., Katritch, V., Boutet, S., Messerschmidt, M., Williams, G. J., Koglin, J. E., Marvin Seibert, M., Klinker, M., Gati, C., Shoeman, R. L., Barty, A., Chapman, H. N., Kirian, R. A., Beyerlein, K. R., Stevens, R. C., Li, D., Shah, S. T. A., Howe, N., Caffrey, M. \& Cherezov, V. (2014). Nat. Commun. 5, 3309.

Weik, M., Ravelli, R. B. G., Kryger, G., McSweeney, S., Raves, M. L., Harel, M., Gros, P., Silman, I., Kroon, J. \& Sussman, J. L. (2000). Proc. Natl Acad. Sci. USA, 97, 623-628.

White, T. A., Kirian, R. A., Martin, A. V., Aquila, A., Nass, K., Barty, A. \& Chapman, H. N. (2012). J. Appl. Cryst. 45, 335-341.

White, T. A., Mariani, V., Brehm, W., Yefanov, O., Barty, A., Beyerlein, K. R., Chervinskii, F., Galli, L., Gati, C., Nakane, T., Tolstikova, A., Yamashita, K., Yoon, C. H., Diederichs, K. \& Chapman, H. N. (2016). J. Appl. Cryst. 49, 680-689.

Wickham, H. (2009). ggplot2: Elegant Graphics for Data Analysis. New York: Springer.

Yamashita, K. et al. (2017). Submitted.

Yamashita, K., Pan, D., Okuda, T., Sugahara, M., Kodan, A., Yamaguchi, T., Murai, T., Gomi, K., Kajiyama, N., Mizohata, E., Suzuki, M., Nango, E., Tono, K., Joti, Y., Kameshima, T., Park, J., Song, C., Hatsui, T., Yabashi, M., Iwata, S., Kato, H., Ago, H., Yamamoto, M. \& Nakatsu, T. (2015). Sci. Rep. 5, 14017.

Zaefferer, S. (2000). J. Appl. Cryst. 33, 10-25.

Zander, U., Bourenkov, G., Popov, A. N., de Sanctis, D., Svensson, O., McCarthy, A. A., Round, E., Gordeliy, V., Mueller-Dieckmann, C. \& Leonard, G. A. (2015). Acta Cryst. D71, 2328-2343.

Zeldin, O. B., Gerstel, M. \& Garman, E. F. (2013). J. Appl. Cryst. 46, $1225-1230$. 